REZENSIONEN

\section{COVID-19. Corona-Gesetzgebung - Gesundheit und Soziales.}

Von Rainer Schlegel, Miriam Meßling und Frank Bockholdt. Verlag C.H. Beck, München 2020, XIII und 395 S., kart., $€ \mathbf{5 9 , 0 0}$.

Die Corona-Pandemie fordert auch die Rechtsordnung und die Jurisprudenz heraus - allein schon deshalb, weil man kaum noch den Überblick behalten kann, was nun das geltende Recht ist. Insbesondere die Verordnungs- und Anordnungsbefugnisse, die $\$ 5$ Abs. 2 des IfSG nun dem Bundesministerium für Gesundheit in der berühmten „epidemischen Lage von nationaler Tragweite“ einräumt, haben ein recht unübersichtliches Normgeflecht entstehen lassen, das durch Parallelstrukturen auf Länderebene noch ergänzt wird.

Vor diesem Hintergrund ist es aller Ehren wert, dass sich der juristische Betrieb gleich aufgemacht hat, um der Normenflut Herr zu werden. Ganz früh war Kluckert (Hrsg.): Das neue Infektionsschutzrecht, auf dem Markt. Auch das grundlegend umgestaltete IfSG hat gleich mehrere neue Kommentierungen erfahren (Kießling und Eckart/ Winkelmüller, Sangs soll dieser Tage erscheinen). Juristische Grundlagenreflexionen sind ebenfalls bereits erhältlich (Kersten/Rixen: Der Verfassungsstaat in der Corona-Krise). Selbst neue Zeitschriften sind gegründet worden („COVID-19 und Recht - COVuR“).

Wie fügt sich nun das Werk „COVID-19. Corona-GesetzgebungGesundheit und Soziales" dreier Sozialrichter, darunter der Präsident des BSG und eine weitere Richterin des BSG, in diese Literatur ein? Zunächst nimmt es eine thematische Beschränkung vor: Es konzentriert sich auf die gesundheits- und sozialrechtlichen Regelungen, die zur Bewältigung der Pandemie erlassen worden sind. Dies ist aber wie schon der Umfang des Werks mit fast 400 Seiten beweist - nicht wenig, da zahlreiche „Corona-Regelungen“ diese Rechtsbereiche berühren: weil sie die Gesundheitsversorgung (inklusive der Pflege) sicherstellen oder die wirtschaftlich-sozialen Folgeschäden der Pandemie ausgleichen oder zumindest abmildern wollen. Nach einem die Rechtsentwicklung der letzten Monate skizzierenden einführenden Überblick werden die Corona-bedingten Veränderungen in den einschlägigen Rechtsbereichen - gerne im Vergleich zum vorherigen „Normalzustand“ - in 19 Kapiteln dargestellt und analysiert. Das gelingt ganz vorzüglich; der Leser wird präzise und gut nachvollziehbar informiert.

Wie fast alle Werke, die jetzt zum Recht (in) der Pandemie erscheinen, liegt der Schwerpunkt auf der transparenten Darstellung des Rechtszustandes; für eine tiefgreifende Analyse, was sich bewährt hat und welche Rechtsprobleme aufgetreten sind, ist es mitten in der Pandemie noch zu früh. Es ist - wie die Autoren einleitend selbst betonen, - eine „Momentaufnahme“ (S. V). Zudem werfen die hier analysierten Regelungen sicherlich Rechtsprobleme auf, haben aber - anders als etliche freiheitseinschränkende Maßnahmen des „Social Distancing“ - bisher noch nicht oder kaum zu Gerichtsverfahren geführt. Auch die Autoren halten sich in ihren Bewertungen zurück; die verfassungsrechtlichen Zweifel (insbesondere an den uferlosen Ermächtigungen des $\$ 5$ Abs. 2 IfSG) werden erwähnt, es wird aber auch viel Verständnis für die Situation des Gesetzgebers gezeigt (vgl. $\$ 1$, Rdnrn. $5 \mathrm{ff}$.)

Die Halbwertszeit derartiger Bücher wird überschaubar sein: Die meisten der behandelten Regelungen sind bis zum 31.3.2021 befristet (vgl. $\$ 5$ Abs. 4 IfSG) und werden dann hoffentlich außer Kraft treten können. Trotzdem werden uns ihre Auswirkungen und ihre Bewertung noch lange beschäftigen. Ein Blick in die „Corona-Gesetzgebung - Gesundheit und Soziales" informiert dabei zuverlässig über das gesundheits- und sozialrechtliche Arsenal, das die Rechtsordnung Mitte des Jahres 2020 gegen das Virus aufgeboten hat.

\section{Stefan Huster}

Prof. Dr. iur. Stefan Huster,

Lehrstuhl für Öffentliches Recht, Sozial- und Gesundheitsrecht und Rechtsphilosophie,

Juristische Fakultät, GD 2/111,

Ruhr-Universität Bochum,

Universitätsstraße 150, 44780 Bochum, Deutschland https://doi.org/10.1007/s00350-020-5642-8

Basiswissen Gesundheitsrecht.

Von Peter Kostorz. Erich Schmidt Verlag Berlin 2020, 158 S., kart. $€ 19,80$

Der Autor des Werkes, Prof. Dr. rer. soc. Peter Kostorz, lehrt am Fachbereich Gesundheit der Fachhochschule Münster Rechtswissenschaften mit den Schwerpunkten Gesundheitsrecht und Bildungsrecht. Er ist in jüngerer Zeit auch mit zahlreichen Veröffentlichungen auf dem Gebiet der Pflege und der Pflegeausbildung hervorgetreten.

Das vorliegende Werk beschränkt sich, wie es im Vorwort heißt, bewusst auf die Vermittlung von Basiswissen zum Gesundheitsrecht. Die im Werk dargestellten Materien des Gesundheitsrechts sind dabei in der Hauptsache das Recht der GKV und der Sozialen Pflegeversicherung sowie der Sozialhilfe, ergänzt um das Recht der Patientenautonomie und der Haftung bei Behandlungs- und Pflegefehlern. Angesichts der Tatsache, dass das Recht der GKV und der Sozialen Pflegeversicherung ganz wesentlich den Gesundheitsschutz und die gesundheitliche Versorgung der Bevölkerung bestimmen, erscheint es als zweckmäßig, diese Kerngebiete des Gesundheitsrechts einem Band zum Basiswissen des Gesundheitsrechts zugrunde zu legen.

Die vier Kapitel des Werkes befassen sich mit dem Begriff und Gegenstand des Gesundheitsrechts (Kapitel 1), den Ansprüchen auf Gesundheitsleistungen (Kapitel 2), der Erbringung von Gesundheitsleistungen (Kapitel 3) und dem Bewirken von Gesundheitsleistungen (Kapitel 4). Allen Abschnitten ist ein kleiner Block mit Literaturhinweisen vorangestellt.

Das Kapitel zum Begriff und Gegenstand des Gesundheitsrechts beginnt mit einem kurzen verfassungsrechtlichen Hinweis zum Verständnis des Rechts aufGesundheit. Dann werden die verschiedenen Begriffsauffassungen zu Umfang und Inhalt des Gesundheitsrechts dargelegt, wobei sich der Verfasser für ein sehr weites Begriffsverständnis ausspricht (S. 15): Das Gesundheitsrecht umfasst demnach die Gesamtheit aller Rechtsvorschriften, die die Aufgaben, Rechte und Pflichten der Akteure bzw. Institutionen im Gesundheitswesen regeln und so das Gesundheitssystem konstituieren, steuern und ordnen und darauf abzielen, die gesundheitliche Versorgung der Bevölkerung bedarfsgerecht, qualitätsorientiert und wirtschaftlich sicherzustellen. Darauf folgt die Darstellung des gesundheitsrechtlichen Dreiecksverhältnisses. Beim Leistungserbringungs- bzw. Behandlungsverhältnis wird auch auf die heimrechtliche Situation im Zusammenhang mit dem Wohn- und Betreuungsvertragsgesetz eingegangen.

Kapitel 2 befasst sich im Rahmen der Ansprüche auf Gesundheitsleistungen mit der GKV und der Sozialen Pflegeversicherung. Diese Materien werden untergliedert in: Anspruchsberechtigter Personenkreis (Versicherter Personenkreis - Versicherungsfälle), Leistungskatalog, Organisation und Finanzierung. Weiter enthält das Kapitel einen Abschnitt zu den leistungsrechtlichen Schnittstellen zwischen Kranken- und Pflegeversicherung. Während der Versicherungsfall der Krankheit eher nur rudimentär beschrieben wird (S. 28), wird der Komplexität des neuen Begriffs der Pflegebedürftigkeit gebührend, auch mit Graphiken, Rechnung getragen (S. 52ff.). Im Abschnitt zur GKV wird der Kreis der versicherten Personen sehr breit dargestellt, was für die grundsätzlich an den Versicherungsschutz in der GKV anknüpfende Soziale Pflegeversicherung dann knapper ausfallen kann. Für beide Leistungszweige werden die Leistungen kurz, aber prägnant beschrieben, bei den SGB XI-Leistungen mit einer Untergliederung in Basisleistungen, Leistungen der häuslichen Pflege, Leistungen der teil- und vollstationären Pflege und Leistungen der Sozialhilfe. Damit wird das mittlerweile etwas unübersichtlich geratene Leistungssystem des SGB XI gut nachvollziehbar aufbereitet. Hilfreich sind auch hier die tabellarischen Leistungsübersichten.

Etwas eigenartig erscheint die Verortung des Versicherungsfalles des Arbeitsunfalles als leistungsausschließender Tatbestand im Verhältnis zu den Leistungen der GKV (S. 29ff.). Immerhin findet so die Gesetzliche Unfallversicherung Eingang in die Behandlung derjenigen Sozialversicherungszweige, die Gesundheitsleistungen vorsehen. Kurz erwähnt wird auch das Verhältnis von Krankheits- und Behinderungsbegriff.

Kapitel 3 beginnt mit der institutionellen und personellen Aufgabenund Arbeitsteilung bei der Erbringung von Gesundheitsleistungen. Unter ersterer werden die Versorgungsebene und Versorgungsbereiche und

Prof. Dr. iur. Gerhard Igl, Universitätsprofessor a.D., Güntherstr. 51, 22087 Hamburg, Deutschland 
die neuen Versorgungsformen verstanden, unter letzterer geht es um die wichtigsten Gesundheitsberufe. In einem Exkurs werden die Delegation und die Substitution ärztlicher Maßnahmen behandelt. In dem weiteren Abschnitt zur Leitungserbringung im System der gesetzlichen Krankenversicherung werden nach der Erörterung der Vertragsarztzulassung und der Einbindung des Vertragsarztes in die Leistungserbringung die Honorierungsfragen behandelt. Die nicht ganz unkomplizierten Strukturen des ärztlichen Vergütungsrechts werden auf nur drei Seiten textlich wie graphisch sehr fassbar dargestellt. Gleiches gilt für die Darstellung der Krankenhausfinanzierung und der Vergütung der Leistungen im Rahmen des SGB XI.

In Kapitel 4 über das Bewirken von Gesundheitsleistungen wird im ersten Abschnitt eine Ubersicht über die verschiedenen Vertragstypen gegeben. Im zweiten Abschnitt wird die Patientenautonomie unter den Gesichtspunkten der Einwilligung und Einwilligungsfähigkeit, der rechtlichen Betreuung, der Vorsorgevollmacht, der Patientenverfügung, sowie der Notfälle, Zwangsmaßnahmen und der Sterbehilfe erörtert. Der letzte Abschnitt ist der Haftung bei Behandlungs- und Pflegefehlern gewidmet.

Zusammenfassend ist zu sagen, dass dieses Werk zum Basiswissen Gesundheitsrecht sehr viel mehr bietet als es die Betitelung mit „Basiswissen" vermuten lässt. Kostorz versteht Basiswissen nicht nur als die Bereitstellung von notwendigem Wissen zu den einzelnen Bereichen des Gesundheitsrechts, sondern stellt sich der Aufgabe, dieses Wissen auf systematischer Grundlage zu vermitteln. Damit wird ein Verständnis von Gesundheitsrecht erreicht, das qualitativ weit über das hinausreicht, was bisher in diesem Format (130 Textseiten!) geboten worden ist. All das geschieht in präziser und immer verständlicher Sprache. Die didaktische Präsentation insbesondere mit Übersichten und Graphiken unterstütz dieses Verständnis.

Mit dem „Basiswissen Gesundheitsrecht“ von Peter Kostorz liegt ein Werk vor, das für alle, die in die Kerngebiete des Gesundheitsrechts einsteigen wollen, eine systematisch angelegte, didaktisch vorbildlich aufbereitete und trotz des geringen Umfangs durchaus detailreiche Wegweisung für dieses Rechtsgebiet bietet, egal, ob es sich um Studierende oder um Personen handelt, die sich beruflich bedingt mit diesem Rechtsgebiet erstmalig befassen wollen.

Gerhard Igl

\section{Gedächtnisschrift für Herbert Tröndle.}

\section{Herausgegeben von Rainer Bleckmann, Gunnar Duttge, Klaus Ferdinand Gärditz, Christian Hillgruber und Thomas Windhöfel. Verlag Duncker \& Humblot, Berlin 2019, 1030 S. geb., $€ 179,90$, als E-Book $€ 161,90$}

Die aus Anlass des 100. Geburtstags erschienene Gedächtnisschrift für Herbert Tröndle enthält 56 Abhandlungen renommierter Autoren, die sich neben allgemeinen Fragen des (Straf-)Rechts vor allem medizinrechtlichen Themen und dort insbesondere dem rechtlichen Schutz des menschlichen Lebens widmen, einem Bereich also, der im Werk Herbert Tröndles einen Schwerpunkt einnimmt. Die Gedächtnisschrift gliedert sich in sieben Abteilungen, von denen drei die Beiträge zu unterschiedlichen Fragen des Lebensschutzes versammeln und eine weitere der Erörterung anderer zumeist ebenfalls eher grundsätzlicher Fragen des Medizinrechts gewidmet ist. Insgesamt hat damit die Mehrheit der Beiträge der Gedächtnisschrift eine medizinrechtliche Thematik.

In der Abteilung ,,Medizinrecht“ erörtert Brettel das Problem der Triage (S. 405-417) und damit ein Thema, das erst im Zuge der aktuellen Corona-Krise die ihm gebührende Aufmerksamkeit erlangt hat. Der Autor weist zu Recht darauf hin, dass die Verteilung medizinischer Hilfeleistung bei zu knappen Ressourcen auch in reichen Gesellschaften keine nur theoretische Fragestellung ist und veranschaulicht die Problematik am Beispiel eines zu einem Unfall gerufenen Notarztes, der am Unfallort nur entweder den Verletzten $\mathrm{O}_{1}$ oder die Verletzten $\mathrm{O}$ und $\mathrm{O}_{3}$ retten kann. Obwohl die Rettung von $\mathrm{O}_{2}$ und $\mathrm{O}_{3}$ fundamentale Rechtspositionen von $\mathrm{O}_{1}$ missachte, sei der h. M. darin beizupflichten, dass eine dahingehende Entscheidung des Notarztes in Ermangelung einer besseren Alternative durch rechtfertigende Pflichtenkollision ge-

Prof. Dr. iur. Helmut Frister,

Lehrstuhl für Strafrecht und Strafprozessrecht,

Heinrich-Heine-Universität Düsseldorf,

Universitätsstraße 1, 40225 Düsseldorf, Deutschland rechtfertigt sei. Problematisch sei aber, ob sich der Notarzt auch anders entscheiden, also zulasten von $\mathrm{O}_{2}$ und $\mathrm{O}_{3}$ den $\mathrm{O}_{1}$ retten dürfe. Brettel legt zu Recht dar, dass die h. M. auf der Grundlage des im Weichenstellerund im Flugzeugabschussfall postulierten Verbots der quantitativen Gewichtung von Menschenleben konsequenterweise auch eine solche Entscheidung für rechtmäßig erachten muss, meldet aber unter Berufung auf ein Optimierungsgebot zumindest Zweifel an, ob dieses Ergebnis richtig sein kann. Die im Zuge der aktuellen Corona-Krise diskutierte Frage, ob bereits behandelten Patienten der Vorrang gegenüber noch nicht behandelten zukommt, wird von Brettel immerhin mit der Bemerkung gestreift, dass die Unterscheidung zwischen Tun und Unterlassen nicht die maßgebliche Differenzierung sein könne. Die abschließende Forderung des Beitrags, die Verteilung knapper medizinischer Ressourcen „,nich zuletzt im Interesse der Helfer" (S. 416) eindeutiger zu regeln, wirkt vor dem Hintergrund der aktuellen Diskussion, in der kein Geringerer als der Deutsche Ethikrat das von der Akademie für Ethik in der Medizin und sechs weiteren medizinischen Fachgesellschaften (m.E. zu Recht) empfohlene Vorgehen, über knappe Beatmungsressourcen nicht nach dem Prioritätsprinzip, sondern nach der Erfolgsaussicht zu entscheiden, als ,objektiv nicht rechtens“ qualifiziert hat, geradezu hellsichtig.

Einen aufgrund des drastischen Organmangels in Deutschland seit vielen Jahren praktizierten Spezialfall der Triage erörtert der in der Abteilung "Todesfeststellung und Transplantationsmedizin“" anzutreffende, nachdenklich geschriebene Beitrag von Eibach (S. 899-907) zur ethischen Problematik der Kriterien Dringlichkeit und Erfolgsaussicht in der Transplantationsmedizin. Eibach schildert auf der Grundlage einer gut dreißigjährigen Erfahrung als Seelsorger an den Bonner Universitätskliniken in sehr eindringlicher Form die bei der Konfrontation mit den Einzelschicksalen deutlich werdenden problematischen Konsequenzen einer lediglich an objektivierbaren medizinischen Kriterien orientierten Organverteilung. Um diese Konsequenzen abzumildern, will er die Organverteilung nicht ,einem abstrakten Prinzip der Gleichbehandlung“ (S. 901) unterwerfen, sondern insbesondere die mitmenschlichen Beziehungen der Menschen und die aus ihnen resultierenden personalen Verantwortlichkeiten, wie z.B. die Verantwortung einer Mutter für ihre noch kleinen Kinder, bei der Vergabe von Organen berücksichtigen. Er erkennt selbst die Gefahr, damit der ,,subjektiven Willkür in der Vergabe von Organen Tür und Tor" (S. 906) zu öffnen, besteht aber darauf, dass zumindest diskutiert werden müsse, ob es nicht möglich sei, für die nicht medizinisch objektivierbaren Aspekte des Lebens Kriterien zu erarbeiten, die vor subjektiver Beliebigkeit bei der Organverteilung schützen. Auch wenn die damit vorgeschlagene Abkehr von dem Prinzip der formalen Gleichheit verfassungsrechtlich sehr problematisch sein dürfte, regt der aus einer langjährigen Konfrontation mit der Lebenswirklichkeit entstandene Beitrag zum Nachdenken über als vermeintlich selbstverständlich vorausgesetzte juristische Gewissheiten an und ist von daher allemal lesenswert

In der Abteilung „Lebensschutz und Lebensende“ beschäftigen sich gleich zwei Beiträge mit dem im März 2017 ergangenen Urteil des BVerwG zur ausnahmsweisen Erteilung einer Erlaubnis zum Erwerb eines Betäubungsmittels zum Zwecke der Selbsttötung (BVerwGE 158, 142). Beide Beiträge sind naturgemäß vor dem erst in diesem Jahr ergangenen Urteil des BVerfG zur Verfassungswidrigkeit des $\$ 217$ StGB (BVerfG, NJW 2020, 905) verfasst, aber deshalb nicht minder interessant zu lesen. In einer Gedächtnisschrift für einen bekennenden „Konservativen“ (vgl die im Geleitwort aufS. 7 zitierte Würdigung Tröndles durch den früheren Präsidenten des BGH Odersky) ist es durchaus bemerkenswert, dass das insbesondere von konservativer Seite heftig kritisierte, aufgrund eines Briefes des Gesundheitsministers von der zuständigen Behörde nicht angewendete und zum Teil sogar als ,,abwegig“ bezeichnete Urteil des BVerwG in beiden Beiträgen gegen die vorgetragene Kritik in Schutz genommen und im Ergebnis als richtig erachtet wird. Der Beitrag des Strafrechtlers Scheinfeld (S. 661-676) setzt sich dabei insbesondere mit dem als Argument gegen das Urteil ins Feld geführten Straftatbestand des $\$ 217$ StGB auseinander und legt überzeugend dar, dass es nicht möglich sei, diesem Tatbestand einen Sinn zuzuschreiben, der vor der Verfassung Bestand haben könne. Das Urteil des BVerfG über diesen Straftatbestand hat ihm Recht gegeben.

Der Beitrag des Staatsrechtlers Sachs (S. 641-660) nimmt das Urteil des BVerwG zum Anlass, aus grundrechtsdogmatischer Sicht über das „Grundrecht auf Selbsttötung“" nachzudenken. Er stimmt zwar der Entscheidung des zu beurteilenden Falles zu, kritisiert aber, dass das BVerwG nicht begründet habe, warum die Möglichkeit zur Umsetzung einer in freier und ernsthaften Selbstbestimmung getroffenen Selbsttötungsentscheidung auf extreme Notlagen begrenzt sein soll. Mit dieser Kritik nimmt er die Feststellung des BVerfG in seinem Urteil zu $\$ 217$ StGB vorweg, das Recht auf ein selbstbestimmtes Sterben sei als Ausdruck personaler Freiheit nicht auf fremddefinierte Situationen und damit insbesondere nicht auf schwere oder unheilbare Krankheitszustände beschränkt. Über das Urteil des BVerfG hinaus führen dagegen die den 